Research Article

\title{
Damage Detection for Large-Scale Grid Structure Based on Virtual Axial Strain
}

\author{
Jian-xin $Y u \mathbb{D}^{1,2}$ and Hui-feng Tan $\mathbb{D}^{1}$ \\ ${ }^{1}$ National Key Laboratory of Science and Technology for National Defense on Advanced Composites in Special Environments, \\ Harbin Institute of Technology, Harbin 150001, China \\ ${ }^{2}$ Center of Analysis and Measurement, Harbin Institute of Technology, Harbin, 150001, China
}

Correspondence should be addressed to Hui-feng Tan; tanhf@hit.edu.cn

Received 22 June 2019; Revised 3 October 2019; Accepted 9 December 2019; Published 13 January 2020

Guest Editor: Franco Concli

Copyright (c) 2020 Jian-xin Yu and Hui-feng Tan. This is an open access article distributed under the Creative Commons Attribution License, which permits unrestricted use, distribution, and reproduction in any medium, provided the original work is properly cited.

To identify the damaged beams in large-scale spatial structure, a damage indicator based on virtual axial strain calculated from mode shape vectors was proposed. The damage detection process was performed based on the dynamic simulation flowchart. Firstly, random signals were used for excitation and the damage was simulated by decreasing beam elasticity modulus. Then, the NEWMARK- $\beta$ precision direct integral method was appreciated for calculating time history response. Finally, the frequency-domain decomposition method only using output response signal was selected for modal parameter estimation. A double-layer grid structure was taken as example for verifying the damage detection method. Results indicate that the proposed indicator was insensitive to environmental noise and capable of localizing multiple damaged members in space structure without the baseline data.

\section{Introduction}

Large-scale lightweight grid structures provide no obstacle view. They are widely used in civil engineering structures, such as sport stadium, exhibition mall, shopping center, and airport roof. However, the catastrophe of this kind of structures caused severe damage to economy and people's death. So it is very important to assess structural health state of space structures and prevent sudden structural failure. Numerous damage detection indicators and damage detection algorithms were proposed to detect, localize, and quantify damage scenarios, and many key structures were instrumented with the structural health monitoring system. Chang et al. [1] reviewed health monitoring of civil infrastructures, and $\mathrm{Ou}$ and $\mathrm{Li}$ [2] reviewed the research and practice of smart sensor networks with health monitoring systems for civil infrastructures in mainland China. Recently, structural health monitoring methods based on structural global vibration were widely accepted both in academic community and engineering community.

With the occurrence of member loss and other physical parameter variations, static and dynamic behaviors of structure change suddenly. So by monitoring global vibration response, the difference of physical parameters could be measured and the damaged location could be identified; the main issue is to propose a proper damage indicator and an effective damage detection algorithm. Recently, structural health monitoring methods based on structural global vibration were widely accepted both in academic community and engineering community. For example, Oyarzo-Vera and Chouw [3] conducted damage identification of unreinforced masonry panels using vibration-based techniques, and the outcomes demonstrated that vibration-based damage detection in unreinforced masonry structures can be satisfactorily performed. Dynamic parameters such as natural frequency, damping ratio, modal shape, strain mode shape, modal strain energy, and flexibility matrix were fully developed for damage detection. Zhao et al. [4] performed structural damage detection by using single natural frequency and the corresponding mode shape; the generalized flexibility matrix of the updated system was obtained; its changes and sensitivity are derived and used to calculate the location and severity of damage. Fan and Qiao [5] reviewed and comparatively studied the vibration-based damage 
identification methods. Furthermore, Farrar et al. [6] summarized the advantages of the vibration-based damage identification method in structural health monitoring.

However, for geometrical symmetry of cyclic spatial structures, the discrepancies between each other are hard to discern, which hinder the application of many damage detection methods. Besides, there is a unique phenomenon called mode jumping, which means structural eigenvector jumps to different modal orders due to the occurrence of damage in geometrical symmetry structures. It is very difficult to compare data collected from the intact state and the damaged one; hence, the damage detection method only using output response signal is in need.

A new damage indicator named "virtual axial strain" is proposed for damage detection and for large-scale space structures. By calculating node displacement vectors from mode shapes to construct strain along the axis and by comparing strains with the adjacent beams to localize the damaged beams based on monitoring the abnormal value of virtual axial strain, here, a user-defined program based on MATLAB/Simulink platform was developed, and a doublelayer grid structure was taken as example to demonstrate its feasibility.

\section{Theoretical Analysis}

2.1. Static Analysis. For beam element subject to either axial tension or axial compression in space truss structure, axial strain is calculated in the following form:

$$
\varepsilon=\frac{\Delta l}{l}=\frac{N}{E A}
$$

where $N, l, E$, and $A$ stand for external force, beam length, elastic modulus, and cross-section area, respectively. Under constant external force, the axial strains of the damaged beams are larger than those of the intact one, which means the axial strain is a good candidate indicator for damage detection. However, the axial strain could not be used directly in damage detection, because it is hard to apply uniform load for all beams, especially for structures in the field application.

2.2. Virtual Axial Strains. A damage indicator named "virtual axial strain" is proposed; its value is calculated based on nodes displacement from mode shapes. Figure 1 shows a beam in the Cartesian coordinate system, where $O$ and $P$ are the two end points of the beam. $\alpha, \beta$, and $\gamma$ are the angles between the direction of beam and $x$-, $y$-, and $z$-axes in the Cartesian coordinate system. $\Delta x$ and $\Delta y$ are the displacement vectors of nodes in the $x, y$, and $z$ directions. $\Delta L$ is the total deformation projected in the beam axial direction. $L$ is the length of the beam.

The virtual axial strain $\bar{\varepsilon}$ is calculated as follows:

$$
\bar{\varepsilon}=\frac{\Delta L}{L}=\frac{\Delta x}{\cos (\alpha)}+\frac{\Delta y}{\cos (\beta)}+\frac{\Delta z}{\cos (\gamma)} .
$$

Considering the $r$ th mode order, where $r=1,2, \ldots, n$ and the corresponding mode shape is $\varphi$. The axial strain of $j$ beam is calculated as follows:

$$
\begin{aligned}
(\bar{\varepsilon})_{j, r}= & \frac{\left(\varphi_{3 p-2, r}-\varphi_{3 o-2, r}\right) / l_{j}}{\cos \left(\alpha_{j}\right)}+\frac{\left(\varphi_{3 p-1, r}-\varphi_{3 o-1, r}\right) / l_{j}}{\cos \left(\beta_{j}\right)} \\
& +\frac{\left(\varphi_{3 p, r}-\varphi_{3 p, r}\right) / l_{j}}{\cos \left(\gamma_{j}\right)},
\end{aligned}
$$

where $l_{j}$ stands for the length of the beam, subscripts $3 p-2$, $3 p-1$, and $3 p$ stand for the displacement vectors of node $p$ in $x, y$, and $z$ directions, and subscripts $3 o-2,3 o-1$, and $3 o$ are the displacement vectors of node $o$ in the $x, y$, and $z$ directions, respectively. For other beams, the virtual axial strains from $r$ th mode shape are expressed as follows:

$$
(\bar{\varepsilon})_{r}=\left(\begin{array}{c}
\frac{\left(\varphi_{3 a-2, r}-\varphi_{3 b-2, r}\right) / l_{1}}{\cos \left(\alpha_{1}\right)} /+\frac{\left(\varphi_{3 a-1, r}-\varphi_{3 b-1, r}\right) / l_{1}}{\cos \left(\beta_{1}\right)}+\frac{\left(\varphi_{3 a, r}-\varphi_{3 b, r}\right) / l_{1}}{\cos \left(\gamma_{1}\right)} \\
\vdots \\
\cos \left(\alpha_{j}\right) \\
\frac{\left(\varphi_{3 c-2, r}-\varphi_{3 d-2, r}\right) / l_{j}}{\cos \left(\beta_{j}\right)}+\frac{\left(\varphi_{3 c-1, r}-\varphi_{3 d-1, r}\right) / l_{j}}{\cos \left(\gamma_{j}\right)}+\frac{\left(\varphi_{3 c, r}-\varphi_{3 d, r}\right) / l_{j}}{\cos \left(\alpha_{n}\right)} \\
\frac{\left(\varphi_{3 e-2, r}-\varphi_{3 f-2, r}\right) / l_{n}}{\cos \left(\beta_{n}\right)}+\frac{\left(\varphi_{3 e-1, r}-\varphi_{3 f-1, r}\right) / l_{n}}{\left(\varphi_{3 e, r}-\varphi_{3 f, r}\right) / l_{n}}
\end{array}\right)=\left(\begin{array}{c}
(\bar{\varepsilon})_{1 r} \\
\vdots \\
(\bar{\varepsilon})_{j r} \\
\vdots \\
(\bar{\varepsilon})_{n r}
\end{array}\right),
$$

where subscripts $1, j$, and $n$ with $l$ stand for different beam numbers and subscripts $a, b, c, d, e$, and $f$ with mode shape $\varphi$ stand for different node numbers. In this way, each virtual axial strain value of the beam is corresponding to the specific beam number. 


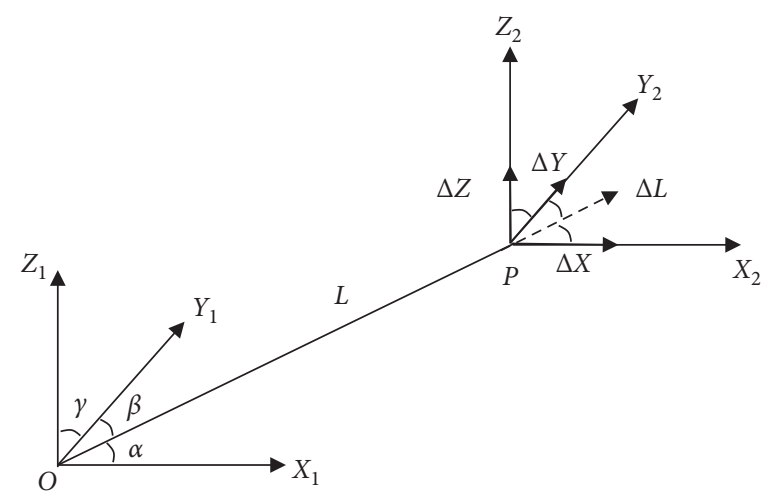

Figure 1: Beam in the Cartesian coordinate system.

2.3. Damage Indicator (DI). After obtaining mode shapes, the virtual axial strain for each beam was calculated based on equation (4). The damaged beams are detected by monitoring the abnormal peak values of virtual axial strains in the following equation:

$$
\mathrm{DI}=\operatorname{abs}\left((\bar{\varepsilon})_{r}\right) .
$$

2.4. Flowchart for Dynamic Simulation Analysis. A flowchart based on user-defined program is proposed for large-scale space structure dynamic simulation analysis. The program is based on MATLAB/Simulink. Figure 2 shows the flowchart for finite element analysis and damage detection dynamic simulation, and the whole flowchart includes the following four steps:

Step 1. Construct the finite element model and solve the eigenequation. Firstly, nodal coordinates and connectivity patterns of members are used to construct the structural finite element model. As each beam element is corresponding to the specific two nodes in space structures, the geometrical model can be obtained based on node coordinates. Then, nodes and beams are modeled with lumped mass element and bar element, respectively. In this program, $A$ denotes the cross-section area of the beam; $N_{\mathrm{n}}$ and $N_{\mathrm{e}}$ denote node matrix and element matrix. Translation degrees of freedom in three directions $(U x, U y$, and $U z)$ for nodes in the supporting boundary are constrained, and the left nodes are free. Obtain the value for elastic modulus $E$, density $\rho$, and passion ratio $\nu$, and then, assemble the element stiffness matrix $\left[k_{i}\right]$ and mass matrix $\left[m_{i}\right]$ to construct the global stiffness matrix $[K]$ and mass matrix $[M]$. Finally, the structural eigenvalue $\lambda$ and eigenvector $\varphi$ are obtained by solving the vibration-governing equation.

Step 2. Calculate dynamic response using the NEWMARK- $\beta$ method. Taking wind load as example, the characteristic of wind time history signal is random signal and could be simulated by white noise, and a low-pass filter is applied to a limit frequency band. Digital signal process parameters including sampling frequency $f_{\mathrm{s}}$, analyzing bandwidth $f_{\mathrm{c}}$, frequency resolution $\mathrm{d} f$, sampling number of point NFFT, sampling interval $\mathrm{d} t$, and sampling time $T$ are defined. Dynamic response is calculated using the NEWMARK- $\beta$ [7] precision direct integral method, and assume the damping matrix $[C]$ is proportional to the stiffness matrix. Here, the proportional coefficient is $\alpha=0.01$ :

(a) The constants for integration are calculated as follows:

$$
\begin{aligned}
& \alpha_{0}=\frac{1}{\gamma \Delta t^{2}}, \\
& \alpha_{1}=\frac{\beta}{\gamma \Delta t}, \\
& \alpha_{2}=\frac{1}{\gamma \Delta t}, \\
& \alpha_{3}=\frac{1}{2 \gamma}-1, \\
& \alpha_{4}=\frac{\beta}{\gamma}-1, \\
& \alpha_{5}=\frac{\Delta t}{2}\left(\frac{\beta}{\gamma}-2\right), \\
& \alpha_{6}=\Delta t(1-\beta), \\
& \alpha_{7}=\beta \Delta t,
\end{aligned}
$$

where $\Delta t$ is the time step and $\beta$ and $\gamma$ are the parameters for the direct integral method.

(b) Calculate the effective stiffness matrix as follows:

$$
[\bar{K}]=[K]+\alpha_{0}[M]+\alpha_{1}[C] .
$$

(c) Calculate the effective force, displacement, acceleration, and velocity at time $t+\Delta t$ as follows:

$$
\begin{aligned}
\{F\}_{t+\Delta t}= & \{F\}_{t+\Delta t}+[M]\left(\alpha_{0}\{u\}_{t}+\alpha_{2}\{\dot{u}\}_{t}+\alpha_{0}\{\ddot{u}\}_{t}\right) \\
& +[C]\left(\alpha_{1}\{u\}_{t}+\alpha_{4}\{\dot{u}\}_{t}+\alpha_{5}\{\ddot{u}\}_{t}\right), \\
{[K]\{u\}_{t+\Delta t}=} & \{F\}_{t+\Delta t}, \\
\{\ddot{u}\}_{t+\Delta t}= & \alpha_{0}\left(\{u\}_{t+\Delta t}-\{u\}_{t}\right)-\alpha_{2}\{\dot{u}\}_{t}-\alpha_{3}\{\ddot{u}\}_{t}, \\
\{\dot{u}\}_{t+\Delta t}= & \{\dot{u}\}_{t}+\alpha_{6}\{\ddot{u}\}_{t}+\alpha_{7}\{\ddot{u}\}_{t+\Delta t},
\end{aligned}
$$




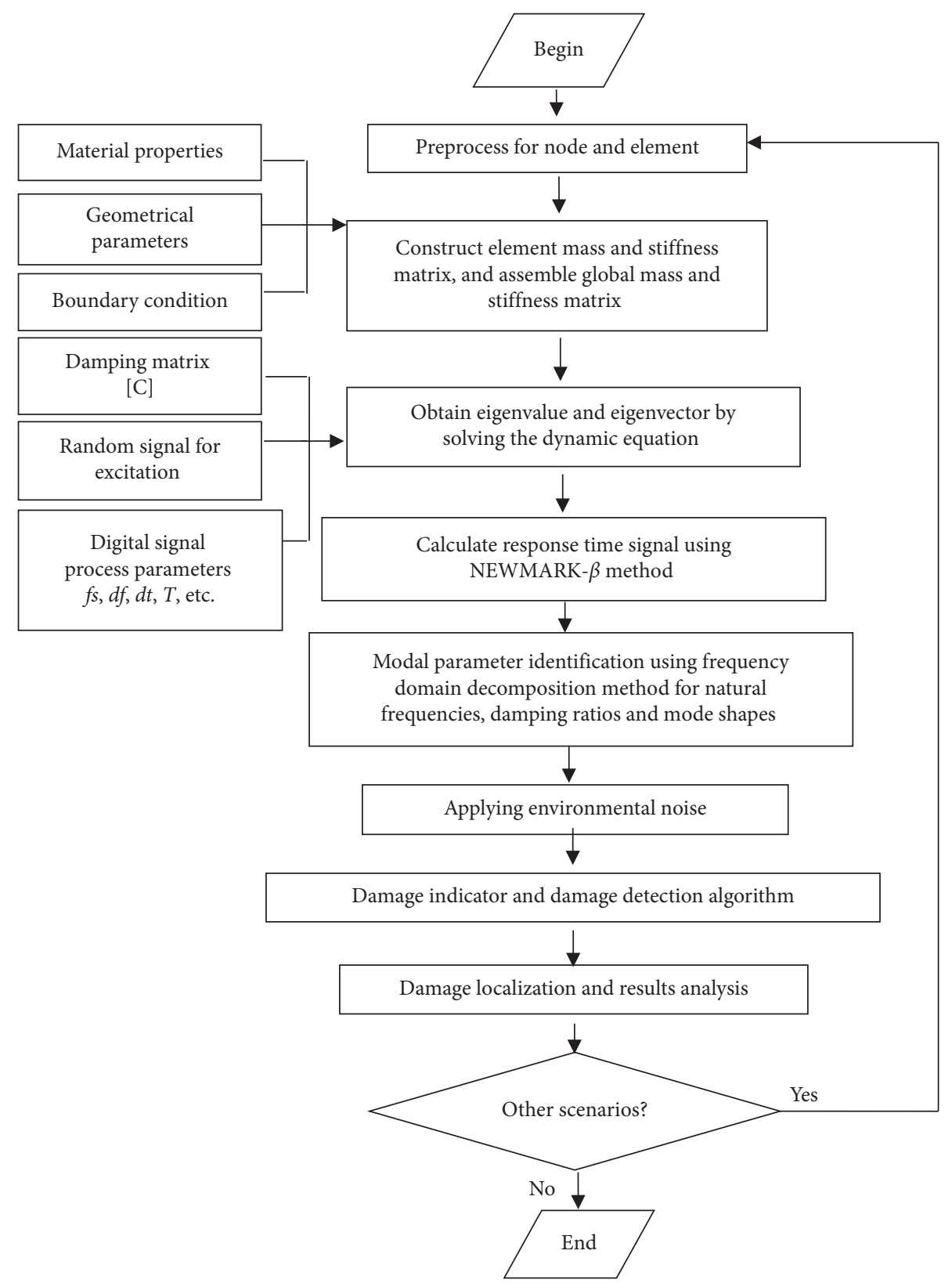

Figure 2: Flowchart for finite element analysis and damage detection dynamic simulation.

where $\{\boldsymbol{u}\}_{0},\{\dot{\boldsymbol{u}}\}_{0}$, and $\{\ddot{\boldsymbol{u}}\}_{0}$ are the displacement, velocity, and acceleration for integration.

Step 3. Estimate modal parameters under environmental noise. Modal parameters are identified based on modal analysis theory. One column or one row number in frequency response function matrix is sufficient for all modal parameters extraction. Here, the frequencydomain decomposition [8] method is selected, because of its capability in separating dense frequencies.

To further validate the effectiveness of the proposed method and simulate the true data from the field test, white noise signal is applied on mode shape:

$$
x(t)^{\prime}=(1+n \% \cdot \operatorname{rand}(N, 1)) \times x(t)
$$

where $x$ and $x^{\prime}$ indicate signal before and after adding white noise signal. $n \%$ denotes noise level, and rand $(N, 1)$ is a function embedded in MATLAB program to generate $N$ random number with a mean of zero and a variance of 1 .

Step 4. Detect damage result analysis based on the damage indicator. After obtaining mode shapes from modal analysis in Step 3, the virtual axial strain is calculated from equation (4) and the damaged beams are detected based on equation (5). 


\section{A Double-Layer Grid Structure}

Figure 3 shows a finite element model of space structure, whose global dimension is $15 \mathrm{~m} \times 15 \mathrm{~m} \times 3 \mathrm{~m}$ with $5 \times 5$ span. The dimension for the top cord, the middle cord, and the bottom cord is $\Phi 285 \times 5 \mathrm{~mm}, \Phi 275 \times 4 \mathrm{~mm}$, and $\Phi 190 \times 3.5 \mathrm{~mm}$, respectively. The material is made of steel, density is $7900 \mathrm{~kg} / \mathrm{m}^{3}$, elastic modulus is $210 \mathrm{GPa}$, and Poisson's ratio is 0.3 . There are 61 nodes, 200 elements, and 123 degrees of freedom. Here, nodes are modeled as a lumped mass with no volume, and all connection points are pin joints and only translational degrees of freedom are considered. For boundary condition, only nodes along the top-layer edge are simply supported and its node displacement in $x, y$, and $z$ directions is constrained. Prestress is considered from self-weight of structure during static analysis, and modal analysis is followed. To evaluate the generality of the proposed damage detection method, beams 17, 88, and 157 from the top, the middle, and the bottom of the space structure were selected and the damaged beams are listed in Table 1. Figures 4 and 5 display the excitation and response time history signal, where the time history is $60 \mathrm{~s}$.

\section{Results and Discussion}

4.1. Effect of Damage on Natural Frequency. Natural frequency reflects structural global vibration characteristics. With the absence of damage, structural natural frequency decreased. Figure 6 shows the natural frequency variance with different damage severities, where $01 E, 03 E, 05 E$, and $07 E$ mean the modulus of the damaged beam are $10 \%, 30 \%, 50 \%$, and $70 \%$ of the intact one. For most mode orders, the frequency discrepancy increased with damage severities. However, the $2^{\text {nd }}$ and $5^{\text {th }}$ mode did not change too much with the severity of damage. These may be due to the fact that the damaged beams are located close to the modal shape nodes of the $2^{\text {nd }}$ and $5^{\text {th }}$ modes coincidently.

The first three mode shapes of intact double-layer spatial structure using finite element analysis are illustrated in Figure 7, where red color means the location of large deformation and blue color means the location of minimal deformation. The first three natural frequencies for the intact model are $6.2152 \mathrm{~Hz}, 6.2152 \mathrm{~Hz}$, and $8.9578 \mathrm{~Hz}$, respectively. The corresponding first three mode shapes for the damaged one are listed in Figure 8 . The first natural frequencies for the damaged model are $6.2097 \mathrm{~Hz}, 6.2150 \mathrm{~Hz}$, and $8.8751 \mathrm{~Hz}$, respectively.

For the intact model, the first and second natural frequencies are the same value due to symmetry. But for the damaged model, the first three natural frequencies are smaller than the intact one, and the sequence of mode shape of the damaged model for the first and the second modes changed compared to the intact one.

4.2. Damage Localization Analysis. Mode shapes are correlated to node coordination, and the damage information is

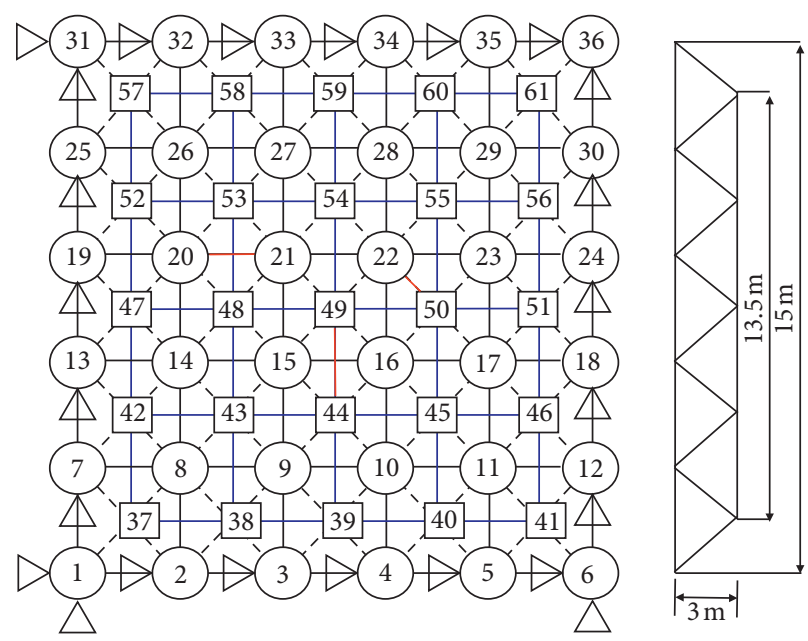

Figure 3: Finite element model of double-layer grid structure.

TABle 1: Damage scenarios.

\begin{tabular}{lcccc}
\hline Element & Location & Node 1 & Node 2 & Severity (\%) \\
\hline 17 & Up cord & 20 & 21 & -50 \\
88 & Low cord & 44 & 49 & -50 \\
157 & Middle cord & 22 & 50 & -50 \\
\hline
\end{tabular}

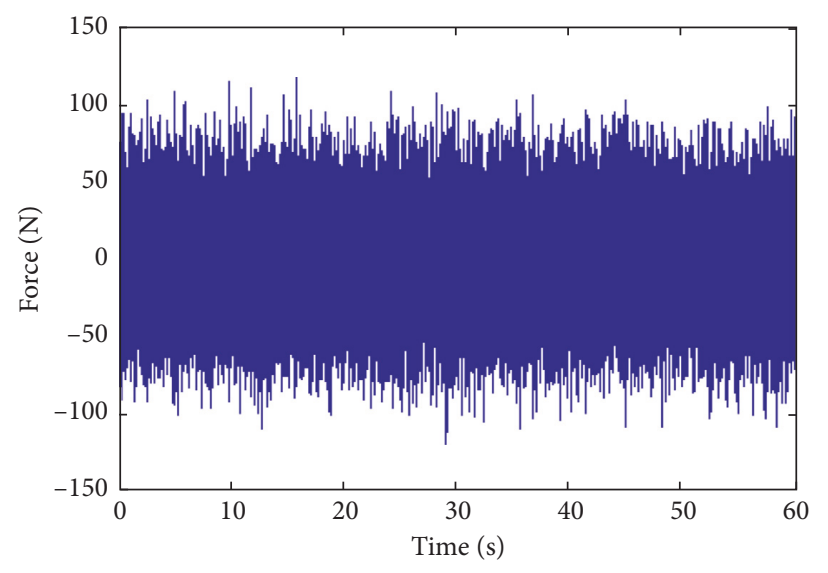

FIgURE 4: Time history for excitation signal.

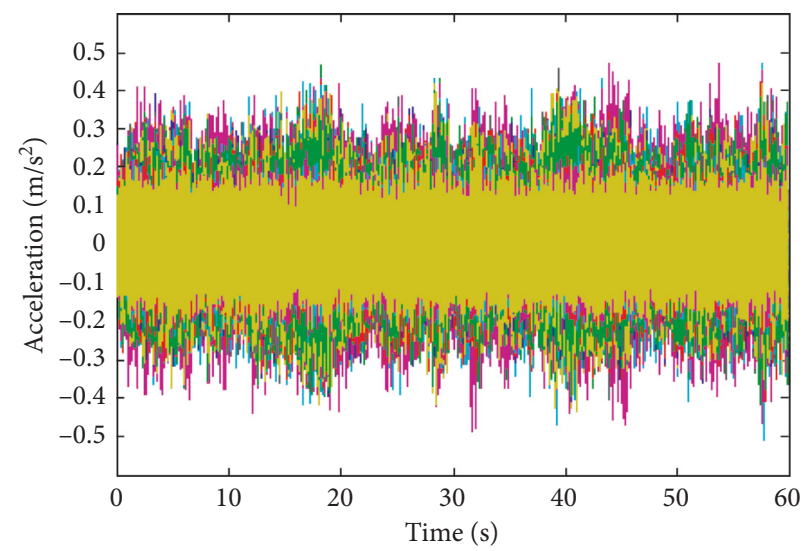

FIgURE 5: Time history for response signal. 


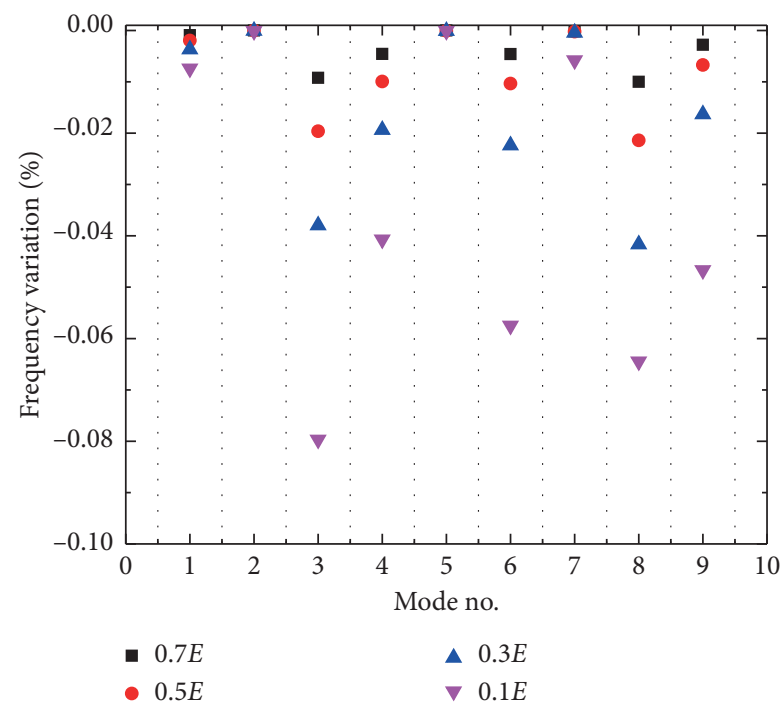

FIGURE 6: Frequency variation under various damage cases.

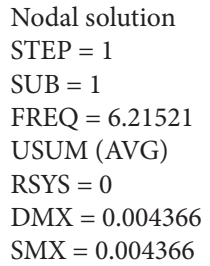

Nodal solution

$\mathrm{STEP}=1$

$\mathrm{SUB}=1$

FREQ $=6.21521$

USUM (AVG)

RSYS $=0$

$\mathrm{DMX}=0.004366$

SMX $=0.004366$
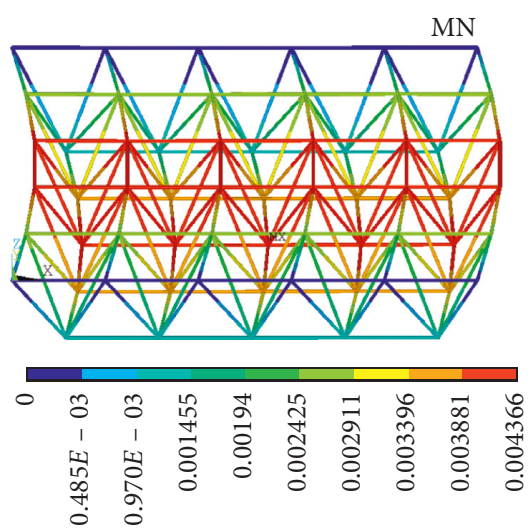

Nodal solution

$\mathrm{STEP}=1$

$\mathrm{SUB}=2$

FREQ $=6.21521$

USUM (AVG)

RSYS $=0$

$\mathrm{DMX}=0.004366$

SMX $=0.004366$

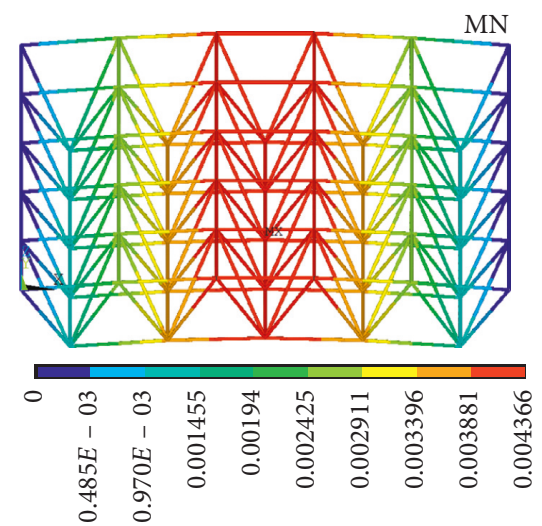

Nodal solution

$\mathrm{STEP}=1$

$\mathrm{SUB}=3$

FREQ $=8.9578$

USUM (AVG)

RSYS $=0$

$\mathrm{DMX}=0.006069$

SMX $=0.006069$

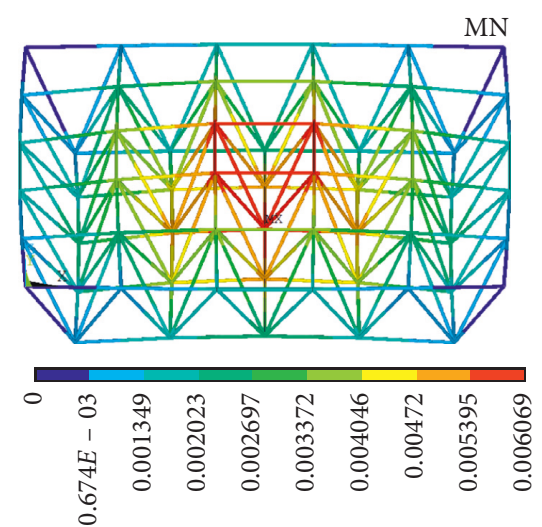

Figure 7: The first three mode shapes for the intact model. (a) $1^{\text {st }}$ order, $6.2152 \mathrm{~Hz}$. (b) $2^{\text {nd }}$ order, $6.2152 \mathrm{~Hz}$. (c) $3^{\text {rd }}$ order, $8.9878 \mathrm{~Hz}$.

contained in mode shapes as well as its deduced components. Figure 9 shows the damage identification results based on the presented virtual axial strain for damage scenarios listed in Table 1, where the damaged beams are identified by monitoring the local peaks and the corresponding damaged beam numbers are 17, 88, and 157 .

The locations correspond to the top cord, the bottom cord, and the middle cord based on nodal coordinates and connectivity patterns of members in the finite element model. Results indicate that the proposed damage indicator is capable of locating damage for multiple damaged beams.
4.3. Damage Sensitivity Analysis. To evaluate the sensitivity of the damage indicator, four different damage severities are investigated, which are $07 E(70 \%), 05 E(50 \%), 03 E(30 \%)$, and $01 E(10 \%)$, respectively. Figure 10 shows the damage identification results with differential damage severities. These sharp peaks clearly indicated the location of beam element, and the amplitude of damage indicator increased with the increasing damage severities. Figure 11 shows that the sensitivities of damage indicator for different beams are a little different; the value of the damage indicator increasing with damage severity shows nonlinearity. These may attribute to the location of the damaged beam and the selected mode shapes. 


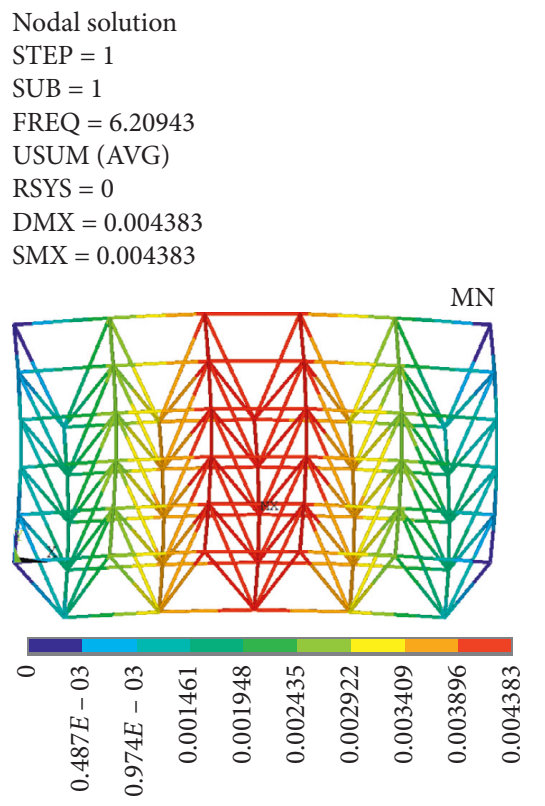

$$
\begin{aligned}
& \text { Nodal solution } \\
& \text { STEP }=1 \\
& \text { SUB }=2 \\
& \text { FREQ }=6.21507 \\
& \text { USUM }(\text { AVG }) \\
& \text { RSYS }=0 \\
& \text { DMX }=0.004369 \\
& \text { SMX }=0.004369
\end{aligned}
$$
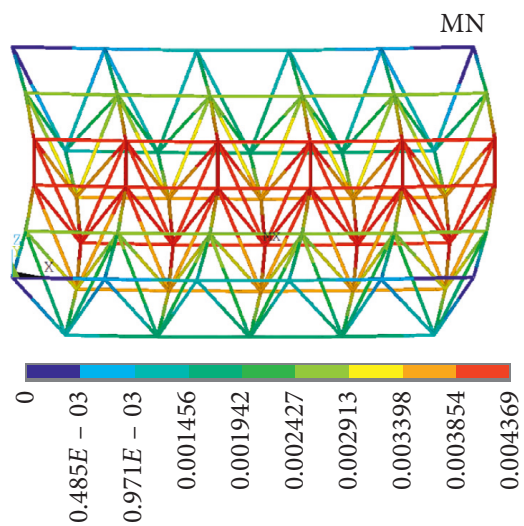

Nodal solution

STEP $=1$

SUB $=3$

$\mathrm{FREQ}=8.87512$

USUM (AVG)

RSYS $=0$

DMX $=0.006071$

SMX $=0.006071$
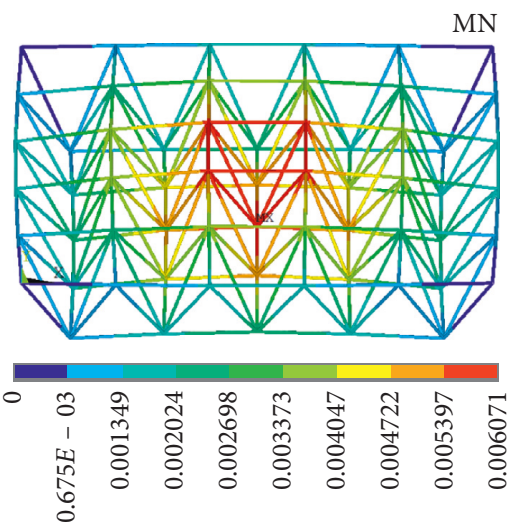

Figure 8: The first three mode shapes for the damaged model. (a) $1^{\text {st }}$ order, $6.2094 \mathrm{~Hz}$. (b) $2^{\text {nd }}$ order, $6.2151 \mathrm{~Hz}$. (c) $3^{\text {rd }}$ order, $8.8751 \mathrm{~Hz}$.

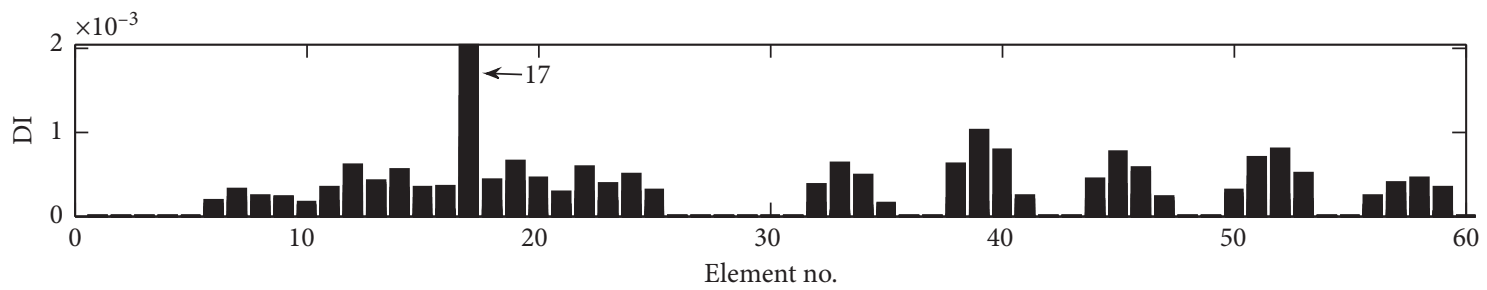

(a)

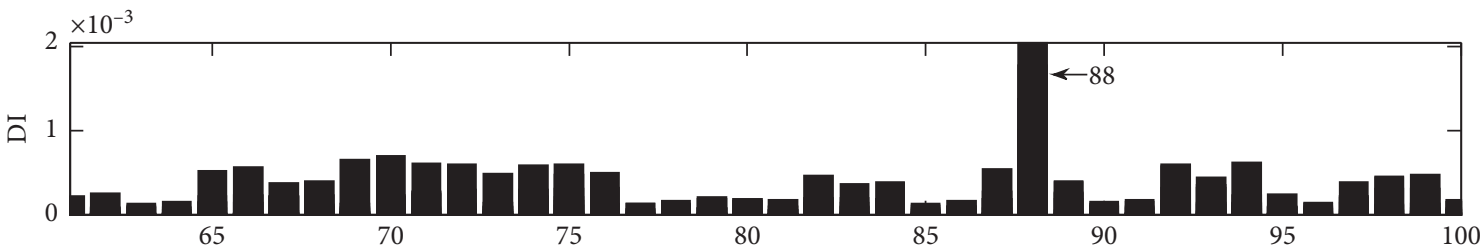

Element no.

(b)

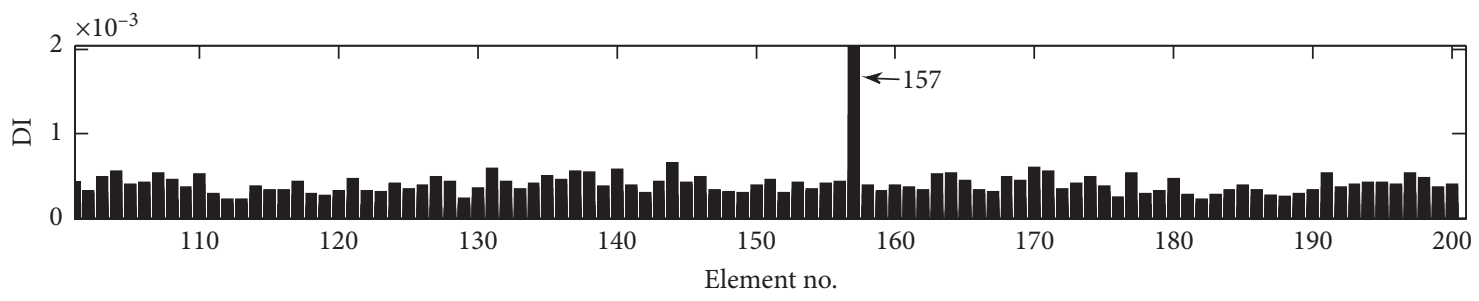

(c)

FIGURE 9: Damage detection results.

4.4. Effect of Environment Noise. Figure 12 shows damage detection results with different noise levels; the damage severity is $03 E(30 \%)$, and the acceleration-time response signal was contaminated with $10 \%$ and $20 \%$ white noise signals. The locations of the damage indicator clearly indicate the location of the damaged beams. However, with the increase of noise level, higher mode shapes should be included in the damage indicator for more accurate damage detection results. 


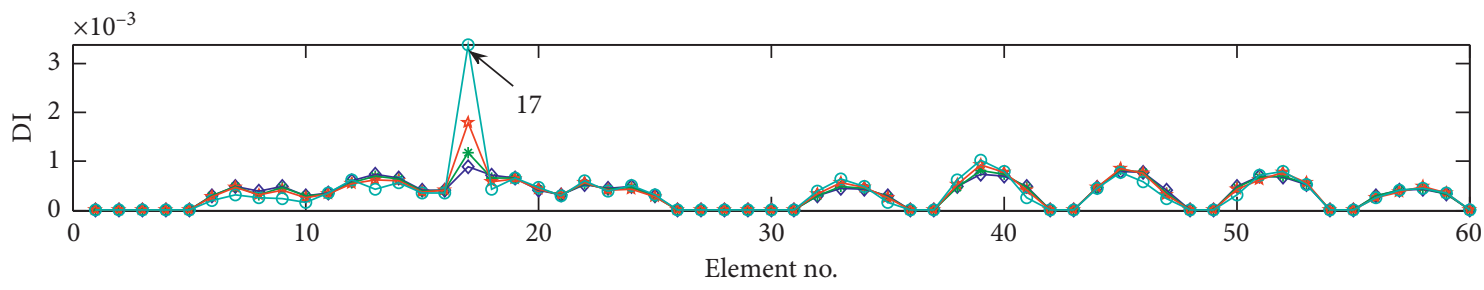
$\diamond 07 E$
$\star 03 E$
* $05 E$
$\circ 01 E$

(a)

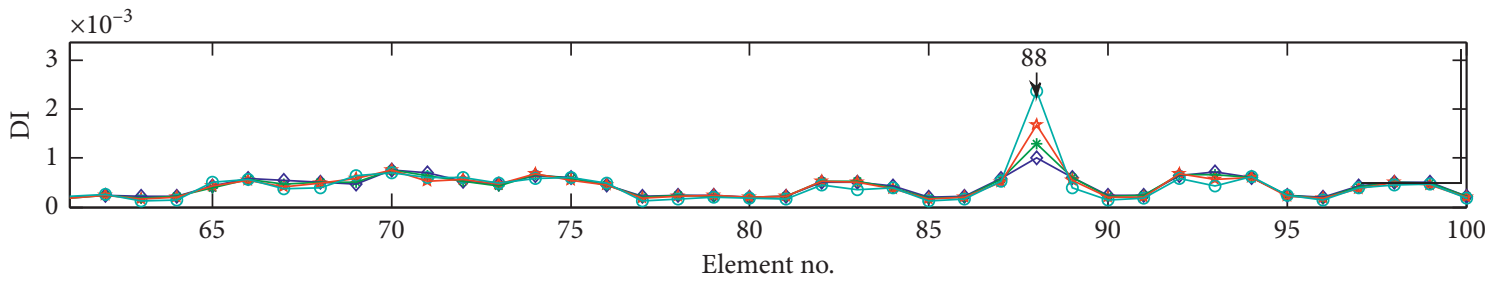
$\diamond 07 E$
屯 $03 E$
* $05 E$
$\circ 01 E$

(b)

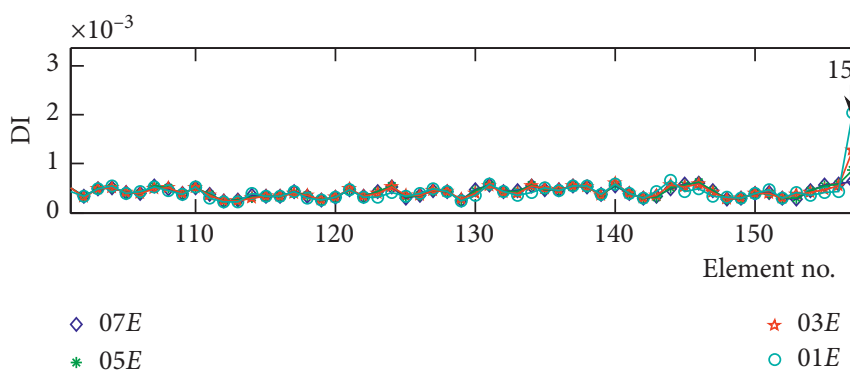

(c)

Figure 10: Damage detection results with different damage severities. (a) For top members. (b) For bottom members. (c) For middle members.

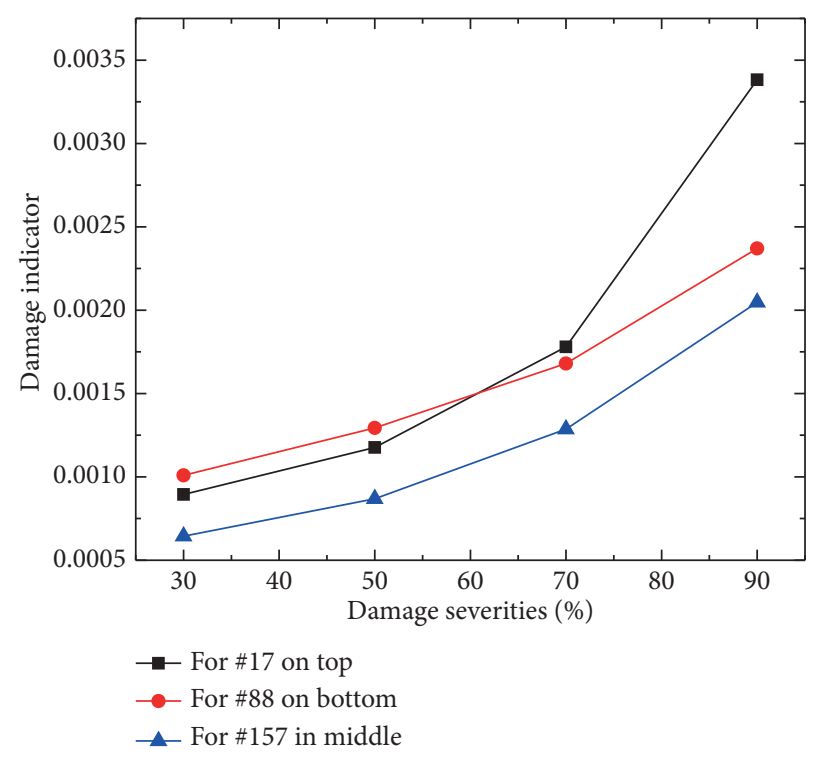

FIgURE 11: Damage indicator with different damage severities. 


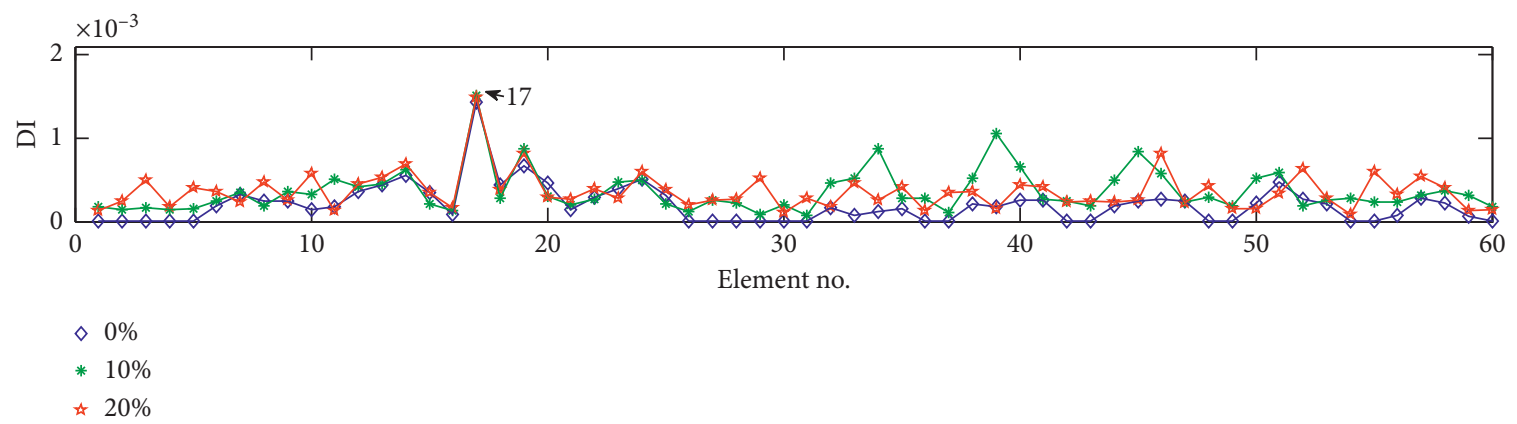

(a)

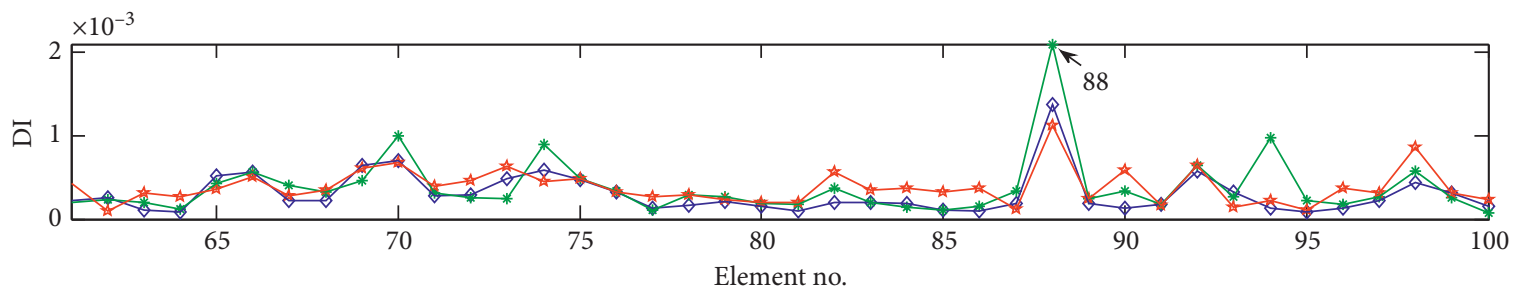

$\diamond 0 \%$

* $10 \%$

+ $20 \%$

(b)

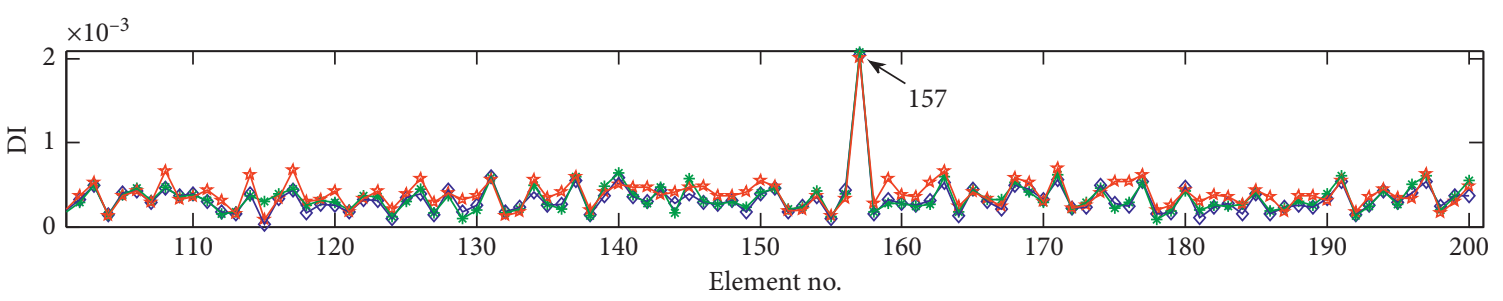

$\diamond 0 \%$

* $10 \%$

$\star 20 \%$

(c)

FIgURE 12: Damage detection results with different noises.

\section{Conclusion}

Damage detection for a double-layer space grid structure was conducted based on the proposed virtual axial strain. The effect of environmental noise with different damage severities was evaluated. Virtual axial strain was computed from mode shape vectors of nodes. By comparing the value of virtual axial strain, the damaged beams were identified. The proposed damage indicator is sensitive to damage severity and insensitive to environmental noise; multiple damaged beams were localized without baseline data.

\section{Data Availability}

The data used to support the findings of this study are available from the first author upon request (contact email: yujianxin03242@163.com).

\section{Conflicts of Interest}

The authors declare that they have no conflicts of interest.

\section{Acknowledgments}

This work received financial support from the Foundation for Innovative Research Groups of the National Natural Science Foundation of China (Grant no. 11421091) and Fundamental Research Funds for the Central Universities (Grant no. HIT.MKSTISP.2016 09), which is gratefully acknowledged.

\section{References}

[1] P. C. Chang, A. Flatau, and S. C. Liu, "Review paper: health monitoring of civil infrastructure," Structural Health Monitoring: An International Journal, vol. 2, no. 3, pp. 257-267, 2003. 
[2] J. P. Ou and H. Li, "Structural health monitoring in mainland China: review and future trends," Structural Health Monitoring, vol. 9, no. 3, pp. 219-231, 2010.

[3] C. Oyarzo-Vera and N. Chouw, "Damage identification of unreinforced masonry panels using vibration-based techniques," Shock and Vibration, vol. 2017, Article ID 9161025, 14 pages, 2017.

[4] B. Zhao, Z. L. Xu, X. N. Kan et al., "Structural damage detection by using single natural frequency and the corresponding mode shape," Shock and Vibration, vol. 2016, Article ID 8194549, 8 pages, 2016.

[5] W. Fan and P. Z. Qiao, "Vibration based damage identification methods: a review and comparative study," Structural Health Monitoring, vol. 10, no. 1, pp. 83-111, 2010.

[6] C. R. Farrar, S. W. Doebling, and D. A. Nix, "Vibration-based structural damage identification," Philosophical Transactions of the Royal Society of London. Series A: Mathematical, Physical and Engineering Sciences, vol. 359, no. 1778, pp. 131-149, 2001.

[7] N. M. Newmark, "A method of computation for structural dynamics," Journal of Engineering Mechanics Division, vol. 85, no. 3, pp. 249-260, 1959.

[8] R. Brincker, L. Zhang, and P. Andersen, "Modal identification of output-only systems using frequency domain decomposition," Smart Materials and Structures, vol. 10, no. 3, pp. 441-445, 2001. 


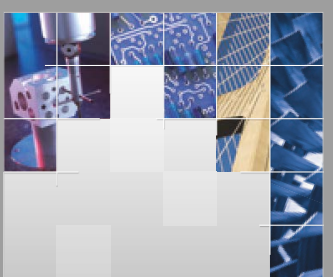

\section{Enfincering}
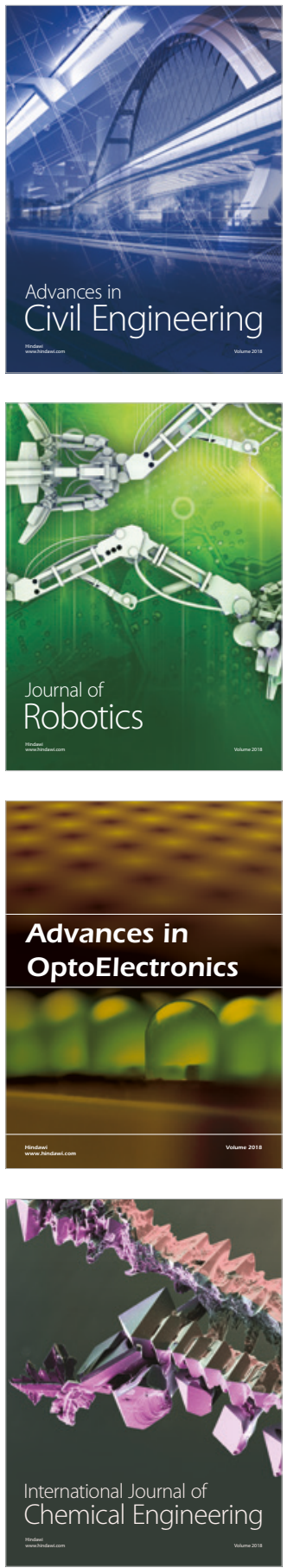

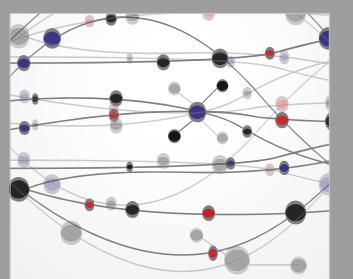

\section{Rotating \\ Machinery}

The Scientific World Journal

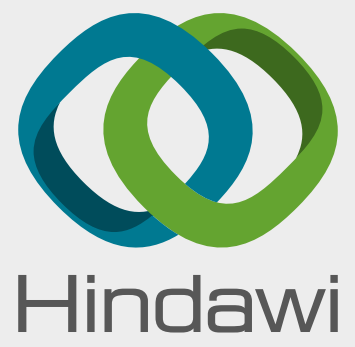

Submit your manuscripts at

www.hindawi.com
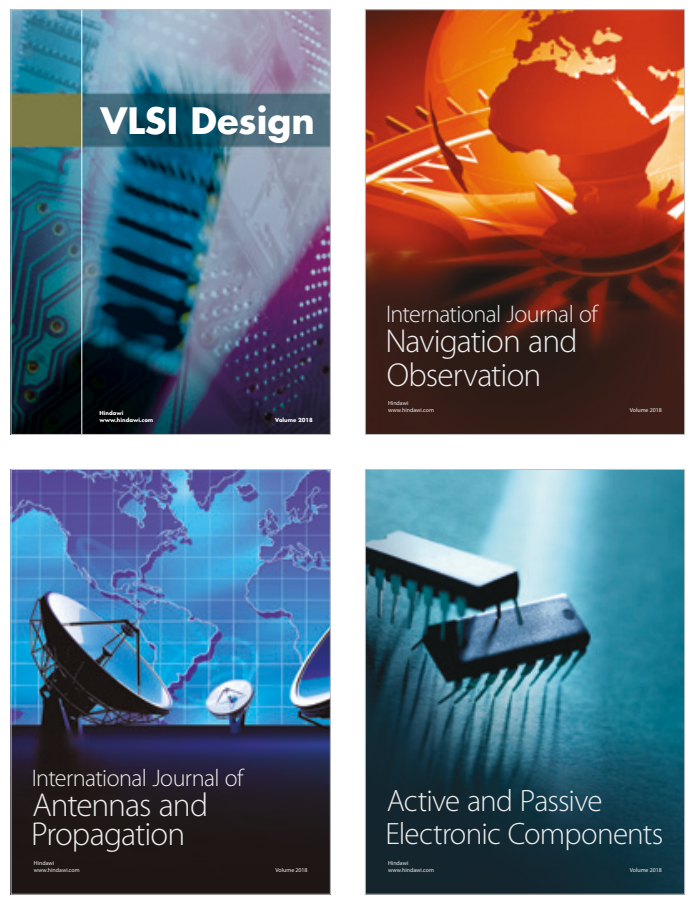
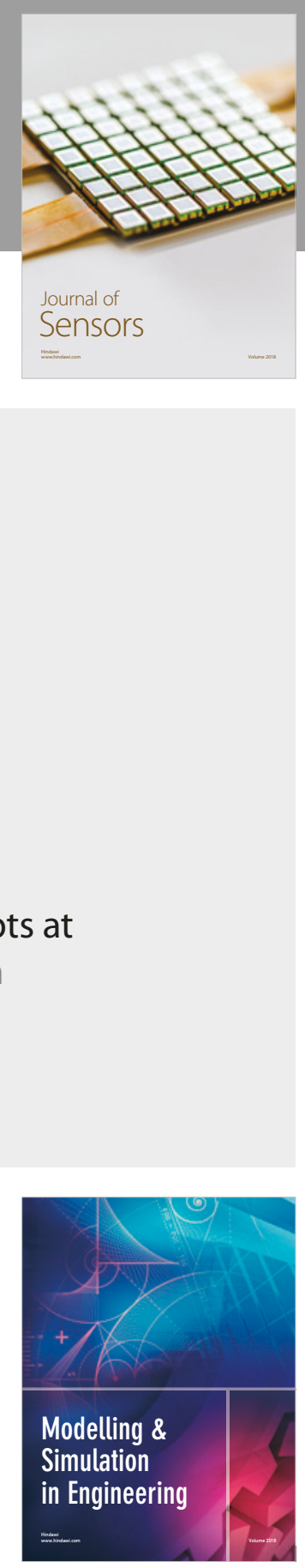

\section{Advances \\ Multimedia}
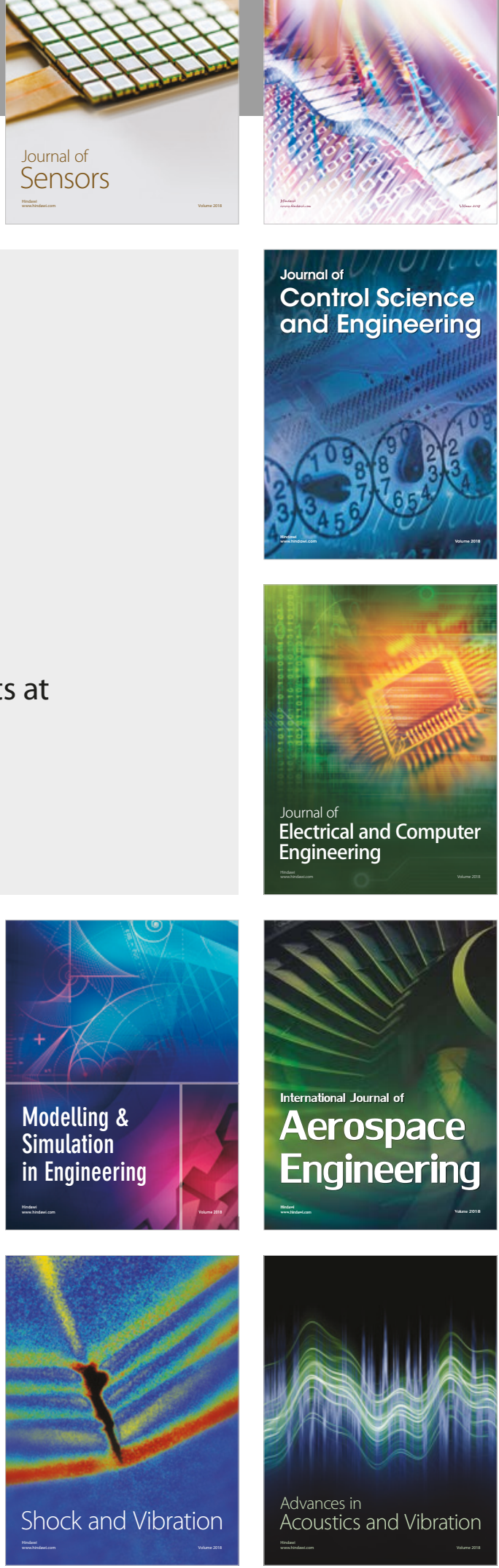